- Multi-professional education has evolved \& adapted over time to reflect key national guidelines \& staff training needs.

- Review of all hospital deaths during April 2018 showed less than $30 \%$ received a continuous subcutaneous infusion of medication prior to death. Clinical indication was clearly documented \& mean starting doses were small (Diamorphine $6 \mathrm{mg}$, midazolam $7.5 \mathrm{mg}$ ). There was no evidence of anticipatory prescribing of syringe drivers, or the prescribing of dose ranges in hospital.

Conclusion A review of end-of-life prescribing prompted by the Gosport Report gives assurance that prescribing practices described, are not seen locally. A culture promoting safe endof-life prescribing has been fostered through readily available, evidence-based guidelines, safe procedures for syringe driver use, wide-reaching multi-professional education co-ordinated by the hospice education centre and an active end-of-life audit group. Strong clinical leadership gives a co-ordinated approach to promoting excellent end-of-life care.

\section{IMPROVING THE PRESCRIPTION OF ANTICIPATORY MEDICATIONS FOR ADULTS RECOGNISED AS DYING IN A DISTRICT GENERAL HOSPITAL}

Harriet Baker, Sue Dargan, Clare Smith. Ashford and St Peter's NHS Foundation Trust

\subsection{6/bmjspcare-2019-ASP.69}

Introduction The NICE quality standards for care of adults in the last hours/days of life state that all patients should have appropriate as required medication (PRN) prescribed for the symptoms (pain/breathlessness, agitation, nausea/vomiting and respiratory secretions) that can occur at the end of life (EoL). This quality improvement project tested the adherence to this in one UK district general hospital.

Aims To investigate whether PRN anticipatory medications were prescribed in accordance to trust guidelines to inform future quality improvement work.

Method This was a prospective audit of 20 consecutive patients across adult wards in June 2018. Drug charts were audited using a tool based on the trust guideline, and the auditor met with junior doctors to discuss concerns around prescribing.

Results $25 \%(n=5)$ of prescriptions were accurately compliant with guidelines. In medications for pain relief and agitation drug dose was correct in 75\% (14) and 60\% (12) respectively, however dose frequency was incorrect in $45 \%$ (9) and $70 \%$ (14), with too long a dose interval in $40 \%$ (8) and 50\% (10) respectively. The PRN medication with the most errors were medication for nausea/vomiting and respiratory secretions with the dose missing or incorrect (no maximum 24 hour dose recommendation) in 95\% (19) and 80\% (16) respectively. Discussion with junior doctors highlighted a lack of awareness of the guidelines.

Conclusions Despite the existence of trust guidelines prescription errors for anticipatory medication at EoL were common. The main concern being patients may have symptoms under treated due missing drugs or too long dose frequency. In collaboration with the specialist palliative care team, pharmacy and junior doctors a poster was created and displayed across all wards. This was supported by teaching sessions delivered by a junior doctor to junior doctors to promote the content of the poster. The adherence to guidelines will be re-audited in 2019 and included in the presentation.
47 DEMONSTRATING SAFE PRESCRIBING AT THE END OF LIFE AFTER THE GOSPORT WAR MEMORIAL HOSPITAL REPORT: A REGIONAL SURVEY OF INPATIENT HOSPICE PRACTICE

Andrew Thorns, David Ward, Georgina Parker, Claire Butler, Habiba Hajallie, Ben Kennedy, Helen McGee. Pilgrims Hospice, Heart of Kent Hospice, Wisdom Hospice, Ellenor Hospice, Hospice in the Weald

\subsection{6/bmjspcare-2019-ASP.70}

Background The Gosport War Memorial Hospital (GWMH) report highlighted significant concerns relating to opioid and sedative prescribing at the end of life. It concluded that the lives of over 450 people were shortened and probably at least another 200 patients were similarly affected. In light of the concern this might raise for the public, the seven hospices in Kent and Medway undertook to compare practice in end of life prescribing and communication.

Methods A medication and record review of patients dying in the in patient units who were admitted for $>24$ hours. To identify patterns of opioid and sedative prescribing and outlying cases for peer review.

Results Continuous subcutaneous infusions (syringe drivers) were used in $70 \%-95 \%$ of patients. Specific explanation to patient or families for their use varied greatly from $20 \%$ $100 \%$. This may in part be due to different interpretation of the standard. Discussion with family that the patient was dying was recorded in $63 \%-100 \%$ of cases. Mean parenteral morphine dose/24 hours varied from 44-141 mg with corresponding medians: $30-60 \mathrm{mg}$. The highest dose used was $930 \mathrm{mg}$.

Mean midazolam doses ranged from 15-34 mg. Median 10-17 mg with the highest dose in 24 hours being $130 \mathrm{mg}$

Levomepromazine was used in less than a third of cases and haloperidol in approximately 10\%.

Phenobarbitone was used once at the final 24 hour dose was $1200 \mathrm{mg}$

Conclusion Whilst our doses are generally comparable with previously published studies, comparing opioid and sedative doses across units enables peer review of the exceptional cases.

Improved communication with patients and relatives about CSCI use and approaching death are needed in some of the units. This survey tool and process offer patients, families and hospice governance structures reassurance that the shocking findings from GWMH are not a risk in these units.

\section{USING ELECTRONIC COMFORT OBSERVATIONS TO SUPPORT THE CARE OF THE DYING ADULT PATIENT - WHAT DO STAFF USERS THINK?}

Nicola Kendal, Richard Cox, Susan Heatley, Ashique Ahamed. Manchester Royal Infirmary, Manchester University Hospitals NHS Foundation Trust

\subsection{6/bmjspcare-2019-ASP.71}

Background The comfort observations is an electronic assessment method that mandates a minimum of 4 hourly checks using parameters that measure comfort (such as pain assessment, mouth care and respiratory effort) rather than physiological measurements, and trigger alerts for escalation of care. It was implemented by the Supportive and Palliative Care Team (SPCT) from October 2017 in the care of dying adult patients trust wide. 
Methods A staff survey was conducted to gain views and user experiences of comfort observations. This paper survey was disseminated to different wards by SPCT members or ward 'end of life care' champions.

Results There were 42 respondents from different wards representing a range of healthcare professionals. Qualitatively the survey showed that staff felt empowered by the comfort observations to proactively monitor dying patients; it enabled me to continue to monitor the patient without causing distress or discomfort'. They facilitated a structured approach and appropriate escalation. Staff felt patient care, and care of loved ones improved as a result; 'Early recognition of pain. Reason for agitation and can be treated without delay. Although EWS is stopped, the continuous monitoring of patient is possible via comfort obs and alert system. Better end of life care experience for patient and relatives'. 100\% of respondents felt the comfort obs were user friendly and would recommend them to other wards.

Conclusions Acute trusts are an important provider of end of life care. Comfort observations support staff to deliver proactive excellent end of life care through routine monitoring of domains of comfort and escalation of care when indicated. A robust training programme to support their use is being planned in addition to conducting a retrospective audit to assess if their use leads to improved frequency of assessing patient and carer needs.

\section{A UK-WIDE SERVICE EVALUATION OF DOCUMENTS RELATING TO ANTICIPATORY PRESCRIBING AT THE END OF LIFE}

Richella Ryan, Anna Spathis, Ben Bowers, Stephen Barclay. University of Cambridge

\subsection{6/bmjspcare-2019-ASP.72}

Background Anticipatory Prescribing (AP), or 'Just in Case' prescribing ahead of need, is a well-established component of end of life care in the UK. It aims to facilitate effective symptom control in the home and care home setting and reduce crisis hospital admissions near the end of life. However, it is a complex multi-system process with great potential for variation and error. The aim of this study is to investigate the role of local policies and procedures in guiding AP practice in community end of life care across the UK.

Methods The first (England) phase of a UK-wide service evaluation has sampled 55 CCGs in England, stratified by NHS local office region, size and level of urbanisation. A range of stakeholders (senior doctors, nurses, pharmacists or CCG managers) were contacted by email and asked to identify and supply the documents used in their areas to regulate and facilitate AP.

Results Responses obtained to date are from 29 of 55 CCG areas in England (53\%). Whilst all areas have some form of generic prescribing guidance, only $11 / 29$ (38\%) have specific AP policies, 10/27 (37\%) have AP-related patient information leaflets and 4/27 (15\%) have AP standard operating procedures.

Conclusion Despite the complexity of the AP process, there is limited formal documentation of standard procedures and professional responsibilities in many areas, with considerable variation in type and level of regulation. Data collection is continuing and will be extended to all four UK countires, with qualitative analysis of responses and documents supplied planned.

\section{0 \\ VOLATILE ORGANIC COMPOUNDS PREDICT THE LAST WEEK OF LIFE IN LUNG CANCER PATIENTS}

S Coyle, E Chapman, S Mason, D Hughes, M Boyd, C Probert, J Ellershaw. St Helen's and Knowsley Hospitals Trust, University of Liverpool, Royal Liverpool and Broadgreen University Hospitals NHS Trust

\subsection{6/bmjspcare-2019-ASP.73}

Background Recognising dying is difficult and is an ongoing difficulty for doctors. We believe there is a process to dying and animal studies support this theory. We previously demonstrated that a number of volatile organic compounds in urine, change in the last weeks and days of life of patients in a small mixed cancer group. We needed to verify this finding in a suitably powered follow-up study.

Method We prospectively collected urine samples from people with lung cancer. We aimed to compare samples from 25 people in each of the last 3 weeks of life to a control group, 50 people with lung cancer who lived 3 or more months from the time of sampling. The urine samples were analysed for volatile organic compounds by gas chromatography mass spectrometry (GC-MS).

Least Absolute Shrinkage and Selection Operator (LASSO) logistic regression was used to analyse the GC-MS data and create a statistical model.

Results We recruited 162 people in total; 29 in the last week; 28 in the second last week; 30 in the third last week of life; 74 controls i.e. samples taken $>3$ months from death; 424 urine samples.

A model was created to predict whether a patient would die within 1 week. It has an optimism corrected AUC of 0.851 (95\% CI: $0.767,0.911$ ); sensitivity 78.6\% (95\% CI: $(64.3 \%, \quad 89.3 \%))$; specificity $83.1 \% \quad(95 \%$ CI: $\quad(69.9 \%$, $92.3 \%))$. The model identified a selection of compounds that contributed to the identification of patients who were close to death.

Discussion

- The results confirm that volatile organic compounds can predict when people with lung cancer are in the last week of life.

- Our model to predict when a person with lung cancer is in the last week of life is approximately $80 \%$ accurate.

\section{HARD RESET INCREASES IDENTIFICATION OF PEOPLE AT THE END OF LIFE}

Carol Scholes, Jane Lawson, Marian Townsend. Herts Community NHS Trust

\subsection{6/bmispcare-2019-ASP.74}

Introduction Recognition that someone is entering the last year of life enables access to Palliative and End of Life Care (PEoLC) including advance care planning. There is currently under-identification of people in the last year of life. The NHS Community Trust covers a population of 1.1 million. Numbers of people identified as being in the last year of life have increased significantly since 2014, with strategies including staff education, use of the 'surprise question' and development and support of a PEoLC Champion in each service. However under-identification continued.

Aim To enable access to PEoLC through improved identification of the patients known to the Trust who are likely to be in the last year of life. 